\title{
Glomerular disease and acute kidney injury in Sudan: Demographics, histological diagnosis and outcome
} \author{
A O Phillips, ${ }^{3} \mathrm{MB} \mathrm{ChB}, \mathrm{BSc}, \mathrm{MD}, \mathrm{FRCP}$ \\ ${ }^{1}$ Nephrology Unit, Armed Forces Teaching Hospital Omdurman, Sudan \\ ${ }^{2}$ Faculty of Medicine, Karary University, Omdurman, Sudan \\ ${ }^{3}$ Institute of Nephrology, Cardiff University School of Medicine, Cardiff, Wales, UK
}

E E A El-hassan, ${ }^{1} \mathrm{MBBS}, \mathrm{MRCP} ; \mathbf{M}$ B Ghalib $,{ }^{2} \mathrm{MBBS}, \mathrm{MRCP} ;$ A I S Ibrahim; ${ }^{1}$ B Phillips, ${ }^{3} \mathrm{MB}$ ChB;

Corresponding author: A O Phillips (phillipsAO@cf.ac.uk)

Background. Acute kidney injury (AKI) is a relatively common clinical condition, associated with high rates of mortality. Although there is extensive literature on the nature and consequence of AKI in the developed world, much less is known in the developing world and more specifically in sub-Saharan Africa (SSA).

Objectives. To describe the demographics, histological diagnosis and clinical course of patients presenting with AKI to a single centre in Sudan.

Methods. Retrospective data were collected on 100 consecutive patients with AKI and an active urinary sediment, who underwent diagnostic native percutaneous renal biopsy.

Results. The mean (standard deviation) age of those biopsied was 33.6 (14.1) years of age, with a preponderance (58\%) of females. The most common clinical indications for biopsy were AKI associated with haematuria and proteinuria (72\%), AKI and proteinuria (22.5\%) or AKI and haematuria (5\%). The frequencies of the most common primary glomerulonephritides (GN) were focal segmental GN (15\%) and mesangiocapillary GN (8\%). Lupus nephritis was the most frequent secondary GN associated with AKI (31\%) and the most common overall histological diagnosis. Peak creatinine, but not oliguria, at presentation predicted likelihood of remaining dialysis-dependent. Age at presentation but not baseline renal function by estimated glomerular filtration rate (eGFR), was associated with the likelihood of having residual chronic kidney disease following an episode of AKI.

Conclusions. The data suggested differences in the pattern of intrinsic renal/glomerular disease leading to AKI to those published and mainly derived from the developed world and patients in SSA.

S Afr Med J 2016;106(7):704-708. DOI:10.7196/SAMJ.2016v106i4.10392

Acute kidney injury (AKI) is a relatively common clinical condition, although the nature of AKI around the world is not well documented. Approximately $90 \%$ of published studies reporting the nature of AKI are from the developed world. ${ }^{[1]}$ This literature suggests that severe AKI requiring renal replacement therapy is associated with high rates of in-hospital mortality. ${ }^{[2]}$ Less severe degrees of kidney injury have also been associated with a significant increased risk of mortality, prolonged duration of hospital stay and associated costs. ${ }^{[3,4]}$ AKI has also been clearly associated with long lasting health consequences for patients. ${ }^{[5]}$

In contrast to the extensive literature on the nature and consequence of AKI in the developed world, there are scant studies focused upon AKI in sub-Saharan Africa (SSA). AKI is now highlighted as a 'global health issue, with a need for more accurate information on the burden of AKI to optimise strategies for prevention and lessening its impact globally. We have previously reported on the nature of AKI in Ethiopia, which focused on a population of patients admitted to a rural hospital through acute medical and surgical routes. ${ }^{[6]}$ These data would suggest that there were differences in both the nature and impact of AKI in this SSA country compared with the studies mainly derived from the developed world. Our previous publication supports the assumption generated from the limited literature from the developing world that AKI in SSA is a disease of the young in whom pre-renal mechanisms predominate, ${ }^{[7,8]}$ as a result of which overall mortality in the context of AKI may be lower. ${ }^{[9,10]}$ Very little is known however regarding the nature of intrinsic renal disease and its association with AKI in this part of the world.
In the current study we have characterised the nature of intrinsic renal disease resulting in AKI in the Sudan. We describe the demographics, histological diagnosis and clinical course of patients presenting to a single centre with AKI and active urinary sediment in whom native percutaneous renal biopsy was performed.

\section{Methods}

This retrospective study of patients undergoing native renal biopsies from January 2005 until December 2014 was conducted in a singlecentre in Sudan to evaluate the pattern of intrinsic renal disease presenting as AKI. Percutaneous renal biopsy specimens were stained and analysed by light microscopy and immunohistochemistry (IgG IgM, IgA and C3). For light microscopy, paraffin sections were routinely stained with periodic-acid Schiff stain, haematoxylineosin, silver-methanamine and Congo red. Patients included in the analysis all presented with AKI defined by creatinine criteria associated with haematuria and RBCs cast or dysmorphic RBCs, and/or proteinuria of more than $1 \mathrm{~g} / \mathrm{d}$. No patients with suspected pre-renal AKI were included. The definition and recommendations provided by the kidney disease improving global outcomes (KDIGO) clinical guidlines for AKI, using creatinine (sCR) criteria were used for identification of AKI. ${ }^{[11]}$

Baseline sCr values for patients were determined through review of all $\mathrm{sCr}$ values taken from the patient during the preceding 12 months. For patients with unknown baseline values, $\mathrm{sCr}$ values charted after AKI resolution, were used to give an approximation of baseline $\mathrm{sCr}$ and confirmation of true AKI (this was applicable 
to 14 patients). Hypertension at the time of AKI presentation was defined as any reading above or equal $140 / 90$ on at least three successive occasions. Oliguria at presentation was defined as a urine output of less than $0.5 \mathrm{~mL} / \mathrm{kg} / \mathrm{h}$ for 6 hours. Patients were classified as having pre-existing chronic kidney disease (CKD) and therefore an episode of acute on chronic renal failure if the baseline eGFR (calculated by the abbreviated four variable MDRD equation) was $<60 \mathrm{~mL} / \mathrm{min}$ according to National Institute for Health and Clinical Excellence (NICE) CKD guidelines. ${ }^{[12]}$ Recovery from AKI was defined as achievement of sCr no longer in keeping with the definition of AKI in comparison to baseline $\mathrm{sCr}$ values, or a return to within the normal range if baseline was not available. CKD was defined as residual renal impairment with persistent eGFR $<60$ but $>15 \mathrm{~mL} / \mathrm{min}$. Dialysis outcome was defined as all patients with post AKI eGFR $\leq 15 \mathrm{~mL} / \mathrm{min}$.

Statistical analysis was carried out using SPSS software, version 20 (SPSS Inc., USA). The Student $t$-test was used for analysis of normally distributed data. Categorical data were compared using a Pearson $\chi^{2}$ test. $p$-values $<0.05$ were considered to represent statistically significant differences.

\section{Results}

\section{Patient characteristics}

Data were collected on 100 consecutive patients with AKI who underwent diagnostic renal biopsy. Basic patient characteristics are shown in Table 1 . The average age of the patients was younger than that generally published in series of patients with AKI in the developed world, with a slight preponderance of female patients. Baseline renal function was unavailable for 30 patients. For the remainder only 9 patients had evidence of pre-existing CKD defined as an eGFR $<60 \mathrm{~mL} / \mathrm{min}$.

Of those with baseline measurement of renal function (70 of 100 ), almost half of the patients had AKI3, with the remainder equally distributed between AKI1 and AKI2. The most common clinical presentation was AKI associated with both haematuria and proteinuria $(72 / 100)$, with the remaining patients presenting with isolated proteinuria $(22 / 100)$ or haematuria (5/100). For the remaining patients, urinalysis was negative for both proteinuira and haematuria, but urine microscopy demonstrated the presence of granular casts. Of the total cohort, 65 patients were hypertensive at presentation, 41 of which presented with AKI, haematuria and proteinuria. In 33 patients AKI diagnosed by creatinine criteria was also accompanied by oliguia at presentation.

\section{Histological diagnosis}

Histological diagnosis for all patients is shown in Table 2. The most common histological diagnosis in this series of patient with AKI was lupus nephritis $(n=31)$. For four of these patients there was no histological classification. Of the remaining patients, 1 staged as class 2 (mesangioproliferative), 4 as class 3 (focal sclerosis), 15 as

\section{Table 1. Patient characteristics at time of presentation*}

\begin{tabular}{ll}
\hline Age (years), mean (SD) & $33.61(14.1)$ \\
Male/female, $n$ & $42: 58$ \\
Baseline creatinine (mg/dL), mean (SD) & $1.01(0.5)$ \\
Baseline eGFR (mL/min), mean (SD) & $91.5(30.7)$ \\
Patients with CKD (eGFR $\leq 60 \mathrm{~mL} / \mathrm{min})$ & 9 of 70 with baseline data \\
$\begin{array}{l}\text { *Baseline data on renal function based either on sCr values taken from the patient during the } \\
\text { preceding 12 months, or for patients with unknown baseline values, sCr values charted after } \\
\text { AKI resolution were available for 70 of the } 100 \text { patients. }\end{array}$
\end{tabular}

class 4 (diffuse proliferative), 4 as class 5 (membranous) and 3 as class 6 (advanced sclerosing nephritis). For 25 of the AKI patients, the histological diagnosis was focal segmental glomerulosclerosis, one of which was associated with a diagnosis of leishmaniasis. For 18 patients, the histological diagnosis was mesangioproliferative/ mesangiocapillary glomerulonephritis, of which one patient had evidence of associated schistosomiasis infection. Seven patients were diagnosed as post infectious glomerulonephritis, with all cases associated with a confirmed rise in ASO titres. Four patients were categorised as rapidly progressive (cresentic) glomerulonephritis, one

Table 2. Biochemical parameters, AKI stage and distribution of clinical syndromes at presentation of $\mathrm{AKI}^{\star}$

\begin{tabular}{|c|c|c|}
\hline $\begin{array}{l}\text { Creatinine at presentation }(\mathrm{mg} / \mathrm{dL}) \text {, } \\
\text { mean }(\mathrm{SD})\end{array}$ & $3.81-3.1$ & \\
\hline $\begin{array}{l}\text { Urea at presentation }(\mathrm{mg} / \mathrm{dL}) \text {, } \\
\text { mean }(\mathrm{SD})\end{array}$ & $99.23(54.00)$ & \\
\hline Presenting with oliguria, $n$ & 33 & \\
\hline AKI1, $n(\%)$ & $18(25.7)$ & \\
\hline AKI $2, n(\%)$ & $19(27.1)$ & \\
\hline \multirow[t]{2}{*}{$\mathrm{AKI} 3, n(\%)$} & $33(47.1)$ & \\
\hline & & $\begin{array}{l}\text { With associated } \\
\text { hypertension }\end{array}$ \\
\hline Haematuria and proteinuria, $n$ & 72 & 41 \\
\hline Isolated proteinuria, $n$ & 22 & 13 \\
\hline Isolated haematuria, $n$ & 5 & 2 \\
\hline
\end{tabular}

Table 3. Distribution of histological diagnosis and number of patients treated with immunosuppression

\begin{tabular}{|c|c|c|}
\hline Histological diagnosis & Patients, $n$ & $\begin{array}{l}\text { Treated with } \\
\text { immunosuppression, } n\end{array}$ \\
\hline Lupus nephritis (all classes) & 31 & 27 \\
\hline $\begin{array}{l}\text { Focal segmental } \\
\text { glomerulosclerosis }\end{array}$ & 25 & 20 \\
\hline $\begin{array}{l}\text { Mesangiocapillary } \\
\text { glomerulonephritis }\end{array}$ & 18 & 13 \\
\hline $\begin{array}{l}\text { Post infections } \\
\text { glomerulonephritis }\end{array}$ & 7 & 4 \\
\hline $\begin{array}{l}\text { RPGN/Cresentic } \\
\text { glomerulonephritis }\end{array}$ & 4 & 4 \\
\hline IgA nephropathy & 3 & 0 \\
\hline $\begin{array}{l}\text { Membranous } \\
\text { glomerulonephritis (non- } \\
\text { lupus related) }\end{array}$ & 2 & 1 \\
\hline Mercury poisoning & 1 & 0 \\
\hline Microscopic polyangiitis & 1 & 0 \\
\hline Renal amyloidosis & 1 & 1 \\
\hline Churg Strauss & 1 & 1 \\
\hline $\begin{array}{l}\text { HIV associated } \\
\text { nephropathy }\end{array}$ & 1 & 0 \\
\hline $\begin{array}{l}\text { Non-specific glomerular } \\
\text { changes }\end{array}$ & 5 & 2 \\
\hline
\end{tabular}


of whom was antineutrophil cytoplasmic antibody (ANCA) positive and three who were antinuclear antibody (ANA) positive.

\section{Outcome}

Data on the clinical outcome were available for 92 of the 100 patients. The mean (standard deviation (SD)) follow-up for this cohort was 4.9 (5.7) months. There were five documented deaths among the whole cohort during the acute episode; no data were available on long-term mortality. For the group with follow-up data, 69 patients were given immunosuppressive treatment during the acute phase (predinisolone \pm cyclophosphamide). In 47 patients, renal function returned to within the normal range (or back to the pre-AKI baseline value), and were classified as recovered. Thirty-one patients developed CKD following the AKI episode, while 14 patients remained dialysis dependent for the duration of the time for which follow-up data was available (Table 4). Recovery in the patients given immunosuppression (and for which outcome data was available) showed no statistical difference to that in the group given no specific therapy ( 38 of 69 v. 13 of $23, p=0.9$ ).

The group in which AKI lead to sustained residual renal impairment and CKD was significantly older than the group who recovered renal function to baseline (Table 5). While a higher baseline creatinine was statistically associated with the likelihood of having residual CKD following an episode of AKI, this relationship did not remain significant when baseline renal function was expressed as eGFR. As the calculation for eGFR takes account of age correction of renal function, this further supports the association of age with outcome rather than renal function per se. For those who remained dialysis dependent, the creatinine was higher than for the other two outcome groups at presentation of the AKI episode, suggesting that the degree

Table 4. Outcome of renal function following AKI episode

\begin{tabular}{|c|c|c|}
\hline Renal outcome & Patients, $n$ & $\begin{array}{l}\text { Length of follow-up (mo), } \\
\text { mean (SD) }\end{array}$ \\
\hline Recovery* & 47 & $4.55(6.10)$ \\
\hline $\begin{array}{l}\text { Chronic renal } \\
\text { impairment }\end{array}$ & 31 & $5.19(5.60)$ \\
\hline Dialysis dependency ${ }^{*}$ & 14 & $5.41(4.20)$ \\
\hline No data available & 8 & \\
\hline \multicolumn{3}{|c|}{$\begin{array}{l}\text { *Recovery }=s C r \text { is no longer in keeping with the definition of AKI in comparison with } \\
\text { baseline } s C r \text { values, or a return to within the normal range if baseline was not available. }\end{array}$} \\
\hline \multicolumn{3}{|c|}{${ }^{\dagger} \mathrm{CKD}=$ residual renal impairment with persistent eGFR $<60$ but $>15 \mathrm{~mL} / \mathrm{min}}$. \\
\hline \multicolumn{3}{|c|}{${ }^{7}$ Dialysis is defined as all post-AKI eGFR $\left.\leq 15 \mathrm{ml} / \mathrm{min}\right)$} \\
\hline
\end{tabular}

of renal injury was the most important predictor of the likelihood of a patient becoming independent of renal replacement therapy.

\section{Discussion}

AKI is increasingly prevalent in both developing and developed countries and is associated with severe morbidity and mortality. Across the world there are differences in epidemiology, clinical characteristics of AKI and availability of therapeutic options.

The limited data generated from the developing world has, in the main, highlighted pre-renal aetiologies. In urban regions it is proposed that AKI is predominantly a hospital-acquired disease occurring mostly in older, critically ill multi-organ failure patients with substantial comorbidity. The main cause in this setting being sepsis, and is often associated with nephrotoxic drugs. ${ }^{[13]}$ In rural areas it is proposed that $\mathrm{AKI}$ is usually community-acquired affecting younger and previously healthy individuals in which AKI is associated with diarrhoeal disease, infectious diseases, animal venoms, septic abortions, dyes and natural medicines. ${ }^{[14]}$ Although there are a handful of studies describing the association between glomerular disease and CKD in Africa, ${ }^{[15-17]}$ in contrast very little data exist on the nature of AKI. Specifically, to our knowledge, no studies have characterised glomerular disease in AKI in this geographical context.

Our data demonstrate that glomerular disease presenting as AKI occurred in a young patient cohort, even accepting that the predicted life expectancy for this region is younger than that seen in the developed world. ${ }^{[18]}$ This preponderance of younger patients is consistent with our previous published studies on AKI among acute hospital admissions in the neighbouring country of Ethiopia. ${ }^{[6]}$ The data also demonstrate a preponderance of females (58\%). Although consistent with data from South Africa (SA), ${ }^{[17]}$ this is in contrast to the distribution of AKI in the developed world which predominantly occurs in elderly male populations. ${ }^{[19-21]}$ The data also suggest that AKI associated with intrinsic renal disease/glomerular injury presents predominantly as AKI3. This is also in keeping with our previous data from Ethiopia in which $48 \%$ of patients presented with AKI3. This is in contrast to studies from the developed world in which milder stages of AKI predominate. ${ }^{[2,23]}$ This may suggest that renal diseases in the developed world present to medical services at later stages, which may in part be related to a lack of appreciation of risk in this geographical setting. ${ }^{[2]}$ This pattern of late presentation has also been seen in the context of CKD in SSA and is likely to reflect accessibility to healthcare services in the region. ${ }^{[17,25]}$ It is also of note that in this study, we have only recruited from a cohort of patients with intrinsic renal disease and thus excluded patients with pre-renal AKI, which is also likely to contribute towards the predominance of

Table 5. Factors influencing clinical outcomes

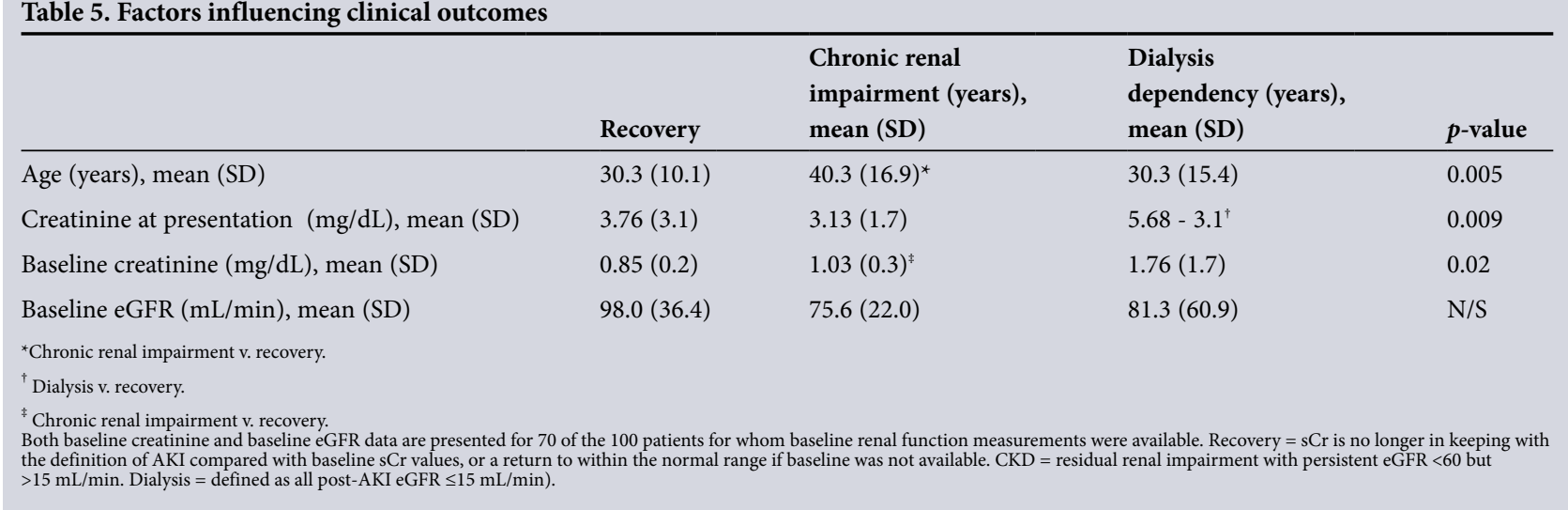


AKI3. Similarly the exclusion of pre-renal causes of AKI accounts for the small number of patients with pre-existing CKD (eGFR $\leq 60 \mathrm{ml} /$ $\min$ ) in this study.

The pattern of glomerular disease presenting as AKI in this study, is also markedly different to that seen in developed countries, but is in keeping with studies describing the patterns of chronic glomerular disease in both the developing world and more specifically SSA. Previous studies from SA suggest that mesangiocapillary glomerulonephritis and focal and segmental glomerulosclerosis are among the most common forms of primary glomerulonephritidis. ${ }^{[17,26]}$ This is also supported by a review of renal histological diagnosis of all renal biopsies reported over 10 years ago from Sudan. ${ }^{[16]}$ Our data supports the assumption that these are also the most common forms of primary glomerular diseases presenting as AKI. It is hypothesised that the differences in prevalence of glomerular diseases may be explained by the 'hygiene hypothesis. ${ }^{\text {'27] }}$ By this hypothesis the overall hygiene and socioeconomic status of a country through exposure to microbes, may predispose its citizens to a Th1-dominant immunological response, which favours the development of glomerulonephritis such as mesangiocapillary glomerulonephritis. In contrast, other types of glomerulonephritis such as IgA nephropathy have a predominantly Th2 response, and better public hygiene and fewer infections observed in industrialised nations my lead to a persistence of the Th2 phenotype. Although we cannot completely rule out the possibility that this pattern of glomeulonephritis may not represent an 'idiopathic' diagnosis, in all but two patients there was no evidence to support secondary causes such as chronic bacterial and viral infections or autoimmune diseases. In the context of the high incidence of FSGS in this study, the identification of coding region variants in apolipoprotein L1 (APOL1), encoding apolipoprotein L1, may be of note. These variants are restricted to populations of recent African descent and are strongly associated with clinically diagnosed primary renal disease/FSGS/nephrosclerosis in the setting of hypertension. ${ }^{[28]}$ Although the frequency of these variants may be lower in eastern African, to our knowledge there is no data on the frequency in Sudan. It is also of note that in the group of patients with FSGS in this study, only $50 \%$ were hypertensive at presentation, possibly suggesting that there is no association in this cohort, although accepting the small numbers affected.

The high incidence of AKI caused by lupus nephritis in this series is also of note. The epidemiology of systemic lupus erythematosus in Africa is largely undetermined. The general view however has prevailed that the incidence of SLE in Africans is negligible. ${ }^{[27]}$ This view has been driven by the rarity of autoimmune diseases in malariaendemic tropical areas has been ascribed to infection-induced immunosuppression. ${ }^{[29]}$ Furthermore, an increased prevalence of SLE has been suggested within people of African descent when they are not living in Africa. ${ }^{[30]}$ This has led to the assumption that on the African continent itself the incidence of SLE is low. It is likely that this view is in part due to the paucity of published studies and likely under-diagnosis resulting from poor access to healthcare. This view on the prevalence of lupus has recently been challenged with a questionnaire-based study across 13 African countries suggesting a much higher than previously anticipated rate of diagnosis. ${ }^{[30]}$ This is also supported by a study from SA in which lupus nephritis represented the most frequent secondary glomerular disease in a retrospective review of biopsy proven renal disease. ${ }^{[17]}$ Our data are therefore consistent with those latter observations and confirms lupus as a significant secondary cause of AKI.

Finally, our study provides intriguing observations related to the acute outcome of AKI caused by intrinsic renal disease. In this patient cohort, peak creatinine as a measure of injury at presentation was associated with increased likelihood of remaining dialysis- dependent, consistent with many published series of AKI. ${ }^{[3,31,32]}$ In contrast to other studies however, oliguria was not associated with outcome. This may be a reflection of the studied group and the exclusion of pre-renal aetiologies in which oliguria may have greater significance. It is also of note that although a higher baseline creatinine was statistically associated with the likelihood of having residual $\mathrm{CKD}$ following an episode of $\mathrm{AKI}$, this relationship did not remain significant when baseline renal function was expressed as eGFR as the main determinant of development of CKD was age at presentation. Clearly in many cases the underlying histological diagnosis represents a 'chronic' condition in which the lesions are not reversible, however how this acute episode impacts on the likelihood of developing progressive CKD is beyond the scope of this study as discussed below. It is also important to note the overall life expectancy in Sudan to place the age of these patients in context.

Although providing useful data on the nature of AKI, our findings need to be qualified by the limitations of the study and the nature of that data that it was possible to collect. This was a single-centre study and its retrospective nature limited the type of data that we could collect. In particular we were unable to collect detailed clinical information regarding patient comorbidities and medication, nor information regarding the presenting episode (i.e. concurrent acute illnesses), which may have contributed to the onset of the AKI episode. In addition the duration of follow-up for the patients due to the nature of the organisation of healthcare did not allow us to evaluate the long-term impact of AKI on either renal function or patient mortality. As such we are unable to assess if there was any delayed improvement or progressive change in renal function for any of these patients. In addition due to the retrospective nature of the study we were unable to identify patients who required renal replacement therapy during the initial illness. Finally, due to the nature of the data storage systems used in the past decade, it is of note that we were unable to collect a complete data set for all patients. Accepting these limitations, this is the first study providing a broad account of biopsy-proven intrinsic renal disease causing AKI, and as such it will help to inform the global discussion regarding AKI.

\section{References}

1. Susantitaphong P, Cruz DN, Cerda J, et al. World incidence of AKI: A meta-analysis. Clin J Am Soc Nephrol 2013;8(9):1482-1493. DOI:10.2215/CJN.00710113

Lameire N, Van Biesen W, Vanholder R. Acute renal failure. Lancet 2005;365(9457):417-430. DOI:10.1016/S0140-6736(05)17831-3

3. Chertow GM, Burdick E, Honour M, Bonventre JV, Bates DW. Acute kidney injury, mortality, length of stay, and costs in hospitalized patients. J Am Soc Nephrol 2005;16(11):3365-3370. DOI:10.1681/ ASN.2004090740

4. Lassnigg A, Schmidlin D, Mouhieddine M, et al. Minimal changes of serum creatinine predict prognosis in patients after cardiothoracic surgery: A prospective cohort study. J Am Soc Nephrol 2004;15(6):1597-1605. DOI:10.1097/01.ASN.0000130340.93930.DD

2004,15(6).1597.1605. DO Co Lafrance JP, Miller DR. Acute kidney injury associates with icr

Nephrol 2010;21(2):345-352. DO1.10.1681/ASN.200906063

Riley S, Diro E, Batchelor P, et al. Renal impairment among acute hospital admissions in a rural thiopian hospital. Nephrology (Carlon) 2013:18(2):92-96. DOI:10.1111/nep.12002

Al-Homrany M. Epidemiology of acute renal failure in hospitalized patients: Experience from southern Saudi Arabia. East Mediterr Health J 2003;9(5-6):1061-1067

8. Jha V, Malhotra HS, Sakhuja V, Chugh KS. Spectrum of hospital-acquired acute renal failure in the developing countries: Chandigarh study. Q J Med 1992;83(4):497-505.

9. Mate-Kole MO, Yeboah ED, Affram RK, Ofori-Adjei D, Adu D. Hemodialysis in the treatment of acute renal failure in tropical Africa: A 20-year review at the Korle Bu Teaching Hospital, Accra. Ren Fail 1996;18(3):517-524

10. Seedat YK, Nathoo BC. Acute renal failure in blacks and Indians in South Africa--comparison after 10 years. Nephron 1993;64(2):198-201.

11. Kellum JA, Lameire N; KDIGO AKI Guideline Work Group. Diagnosis, evaluation, and management of acute kidney injury: A KDIGO summary (Part 1). Crit Care 2013;17(1):204. DOI:10.1186/cc11454

12. National Intitute for Health and Care Excellence. Early identification and management of chronic kidney disease in adults in primary and secondary care, 2008. http://wwwniceorguk/CG73 (accessed September 2008).

13. Santos WJ, Zanetta DM, Pires AC, et al. Patients with ischaemic, mixed and nephrotoxic acute tubular necrosis in the intensive care unit - a homogeneous population? Crit Care 2006;10(2):R68. DOI:10.1186/cc4904

14. Naicker S, Aboud O, Gharbi MB. Epidemiology of acute kidney injury in Africa. Semin Nephrol 2008;28(4):348-353. DOI:10.1016/j.semnephrol.2008.04.003

15. Okpechi IG, Dlamini TA, Duffield M, et al. Outcome of patients with primary immune-complex type mesangiocapillary glomerulonephritis (MCGN) in Cape Town South Africa. PLoS One 2014;9(11):el13302. DOI:10.1371/journal.pone.0113302 
16. Khalifa EH, Kaballo BG, Suleiman SM, Khalil EA, El-Hassan AM. Pattern of glomerulonephritis in Sudan: Histopathological and immunofluorescence study. Saudi J Kidney Dis Transpl 2004;15(2):176-179.

17. Okpechi I, Swanepoel C, Duffield M, et al. Patterns of renal disease in Cape Town South Africa: A 10year review of a single-centre renal biopsy database. Nephrol Dial Transplant 2011;26(6):1853-1861. DOI.10.1093/ndt/gfa655

18. Mokdad AH, Jaber S, Aziz MI, et al. The state of health in the Arab world, 1990-2010: An analysis of the burden of diseases, injuries, and risk factors. Lancet 2014;383(9914):309-320. DOI:10.1016/S01406736(13)62189-3

19. Hsu CY, McCulloch CE, Fan D, et al. Community-based incidence of acute renal failure. Kidney Int 2007;72(2):208-212. DOI:10.1038/sj.ki.5002297

20. Liangos O, Wald R, O'Bell JW, et al. Epidemiology and outcomes of acute renal failure in hospitalized patients: A national survey. Clin J Am Soc Nephrol 2006;1(1):43-51. DOI:10.2215/CJN.00220605

21. Meier P, Bonfils RM, Vogt B, Burnand B, Burnier M. Referral patterns and outcomes in noncritically ill patients with hospital-acquired acute kidney injury. Clin J Am Soc Nephrol 2011;6(9):2215-2225. DOI:10.2215/CJN.01880211

22. Talabani B, Zouwail S, Pyart RD, et al. Epidemiology and outcome of community-acquired acute kidney injury. Nephrology (Carlton) 2014;19(5):282-287. DOI:10.1111/nep.12221

23. Wonnacott A, Meran S, Amphlett B, Talabani B, Phillips A. Epidemiology and outcomes in community-acquired versus hospital-acquired AKI. Clin J Am Soc Nephrol 2014;9(6):1007-1014.
com community-acquired versus

24. Phillips LA, Allen N, Phillips BM, et al. Risk awareness of acute renal injury in three teaching hospitals 4. Phillips LA, Allen N, Phillips BM, et al. Risk awareness of acute renal inju
in Ethiopia. S Afr Med J 2013;103(6):413-418. DOI:10.7196/SAMJ.6424
25. Van Rensburg BW, van Staden AM, Rossouw GJ, Joubert G. The profile of adult nephrology patients admitted to the Renal Unit of the Universitas Tertiary Hospital in Bloemfontein, South Africa from 1997 to 2006. Nephrol Dial Transplant 2010;25(3):820-824. DOI:10.1093/ndt/gfp535

26. Thomas DB, Franceschini N, Hogan SL, et al. Clinical and pathologic characteristics of focal segmental glomerulosclerosis pathologic variants. Kidney Int 2006;69(5):920-926. DOI:10.1038/sj.ki.5000160
gitics

27. Hurtado A, Johnson RJ. Hygiene hypothesis and prevalence of glomerulonephritis. Kidney Int Suppl 2005(97):S62-67.

28. Kopp JB. Rethinking hypertensive kidney disease: arterionephrosclerosis as a genetic, metabolic, and inflammatory disorder. Curr Opin Nephrol Hypertens 2013;22(3):266-272. DOI:10.1097/ MNH.0b013e3283600f8c

29. Symmons DP. Frequency of lupus in people of African origin. Lupus 1995;4(3):176-178.

30. Bae SC, Fraser P, Liang MH. The epidemiology of systemic lupus erythematosus in population of African ancestry: A critical review of the 'prevalence gradient hypothesis'. Arthritis Rheum 1998;41(12):2091-2099. DOI:10.1002/1529-0131(199812)

31. Wang HE, Muntner P, Chertow GM, Warnock DG. Acute kidney injury and mortality in hospitalized patients. Am J Nephrol 2012;35(4):349-355. DOI:10.1159/000337487

32. Xu X, Nie S, Liu Z, et al. Epidemiology and Clinical Correlates of AKI in Chinese Hospitalized Adults. Clin J Am Soc Nephrol 2015;10(9):1510-1518. DOI:10.2215/CIN.02140215

Accepted 24 March 2016 